\title{
PURIFICATION FACTOR CHARACTERIZATION OF ZONE REFINING
}

\author{
BY \\ LOUIS GOLD ${ }^{1}$ AND VIRGINIA JOHNSON ${ }^{2}$ \\ ABSTRACT
}

\begin{abstract}
A quantity $\pi r$, termed the purification factor, defines the potential state of purity realizable in a specimen that has undergone zone-refinement treatment. An exact analysis appears intractable, but neglect of the terminal zone perturbation (which becomes vanishingly small for the infinite ingot) allows a lower bound to be established on the remanent solute impurity. Such numerical values of $\pi r$ are evaluated for some sensible distribution coefficients and zone dimensions involving a maximum of ten passes. An original expression for the solute impurity distribution is deduced and numerically appraised for a range of operational conditions not presentiy tabulated; the relation has been applied to the indicated $\pi_{T}$ determinations.
\end{abstract}

INTRODUCTION

The purpose here is to describe in some detail a useful parameter for characterizing the progress made at any stage of the zone-refining process. In doing so, it may also be of value to present still another manner for expressing the impurity distribution which evidently lends itself more readily to numerical calculation. Thus a quantity identified as a purification factor is explicitly evaluated for a range of conditions considered to be representative of zone-refining practice.

Actually, the present treatment is restricted to an upper bound identification of the degree of purification attained during the refinement precedure; analytic treatment of the terminal zone effect does not appear to be tractable, although the correction could be numerically evaluated. The technique of terminal zone cropping tends to moderate the departure from the ideal purification factor since it operates to offset the approach to an equilibrium impurity distribution. A brief description of this approach was offered earlier $(1){ }^{3}$

\section{IMPURITY BALANCE AND THE PURIFICATION FACTOR}

A simple impurity balance may be posed ignoring the terminal zone perturbation. Conservation of solute in an ingot of length $L$ having an initial distribution $C_{0}(x)$ may be represented as follows:

$$
\sum S=C_{0} L=S_{T}=S_{I, n}+S_{I I, n}+S_{I I, n-1}+\sum_{n-1} S_{j, n-1}
$$

${ }^{1}$ Project Matterhorn, Princeton University, Princeton, N. J.; at present, Radiation Laboratory and Department of Nuclear Engineering, University of Michigan, Ann Arbor. Mich.

2 Lincoln Laboratory, Massachusetts Institute of Technology, Lexington, Mass.

${ }^{3}$ The boldface numbers in parentheses refer to the references appended to this paper. 
where the total impurity solute $S_{T}$ is expressed in terms of the components $S_{I, n}$, the solute in the purified zone after the $n^{\text {th }}$ pass, $S_{I I, n}$, the solute in the liquid zone, $S_{I I I, n-1}$, the solute in the remaining ingot from the previous pass, exclusive of the terminal zone, which must be summed over $S_{f, n-1}$ for the maximum accumulation of impurity after $n-1$ passes.

The compartmental distribution of impurity then is prescribed by the relations

$$
\begin{aligned}
S_{I, n} & =\int_{0}^{x} C_{n}(x) d x \\
S_{I I, n} & =\frac{l}{k} C_{n}(x) \\
S_{I I I, n-1} & =\int_{x+l}^{L-l} C_{n-1}(x) d x \\
S_{f, n-1} & =\left.l C_{n-1}(x)\right|_{x=L-l}
\end{aligned}
$$

where $l$ is the zone length and $k$ is the solute distribution coefficient.

Thus for the fraction of solute impurity removed after the $n^{\text {th }}$ pass $P_{n}$ there derives

$$
P_{n}=\frac{1}{C_{0} L} \int_{0}^{L^{-}-l}\left[C_{n-1}(x)-C_{n}(x)\right] d x
$$

whereas for the cumulative removal of impurity it follows that

$$
P_{T}=\sum_{n} P_{n}=\left(C_{0} L-\int_{0}^{L-1} C_{n}(x) d x\right)\left(C_{0} L\right)^{-1} .
$$

The fraction of impurity yet to be transferred to the terminal zone may be defined as the purification factor $\pi_{T}$ and is clearly the result

$$
\pi_{T}=1-P_{T} .
$$

Explicit calculation of the purification factor demands identification of $C_{n}(x)$ and the subsequent evaluation of Eq. 4 ; the next section deals with this aspect.

ELABORATION OF THE $P_{T}$ RELATION : A NEW FORM FOR $C_{n}(X)$

The detailed calculations of the purification factors are based upon a form of $C_{n}(x)$ not previously described in the literature (2). Starting with the underlying equation (3) which specifies the spatial distribution

4 The usual idealizations are embodied as, for example, perfect mixing of components in the liquidus, limited diffusion in the solidus, etc. 
of solute

$$
\frac{d S_{n}(x)}{d x}=C_{(n-1)}(x)-\frac{k}{l} S_{n}(x)
$$

the solution may readily be shown to be of the form

$$
S_{n}(x)=e^{-(k / h) r}\left\{\int_{0}^{x} C_{(n-1)} e^{(k / h) x} d x+K_{(n-1)}\right\}
$$

wherein the $K_{(n-1)}$ are integration constants prescribed by the initial condition

$$
\left.C_{n}(x)\right|_{x=0}=k \bar{c}, \quad \bar{c}=\frac{1}{l} \int_{11}^{l} C_{(n-1)}(x) d x
$$

when utilized in conjunction with

$$
C_{n}(x)=\frac{k}{l} S_{n}(x)
$$

In the appendix are provided details for the inductive prescription of $Q_{n}(x)$ in the generalized form of $C_{n}(x)$ which resembles that of the single pass result:

with

$$
C_{n}(x) / C_{0}=1-Q_{n}(1-k) e^{-(k / l) x}
$$

$$
Q_{n}=\sum_{p=n}^{1} A_{p} \frac{\left(\frac{k}{l} x\right)^{n-1,}}{(n-p) !}
$$

and the diverse $A_{p}$ in turn given by

$$
\begin{aligned}
A_{3}=1-\frac{A_{j-1} S_{1}}{0 !} & -\frac{A_{j-2} S_{2}}{1 !}-\frac{A_{j-3} S_{3}}{2 !}-\cdots \\
& -\frac{A_{2} S_{j-2}}{(j-3) !}+\frac{1}{(j-2) !}\left(\frac{k}{l}\right)^{j-1} \int_{0}^{l} x^{j-2} e^{-(k / l) x} d x .
\end{aligned}
$$

In $(10 c)$ the $S_{m}$ takes on values determined from

$$
S_{m}=-\int_{0}^{k} x^{j-1} e^{-x} d x=-\left[\int k^{j-1} e^{-k} d k+(j-1) !\right] .
$$

Typically, for $j=1, A_{1}=1$ and $Q_{1}=1$.

In Table I are collected the solute distributions $C_{n}(x) / C_{0}$ as a function of $x / l$ for $n=1$ to 10 with $k$ values selected as $0.001,0.007$, $0.05,0.07$ and 0.12 . The over-all behavior of the solute distribution is exhibited in Figs. 1-6; two different ranges are displayed in Figs. 1 and 2 , with the latter appropriate for small $x / l$ and $k=0.001$. Other values 


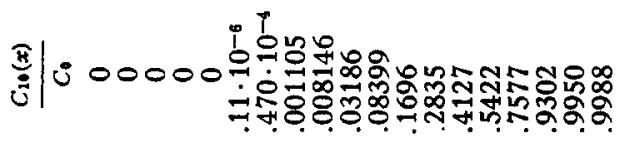

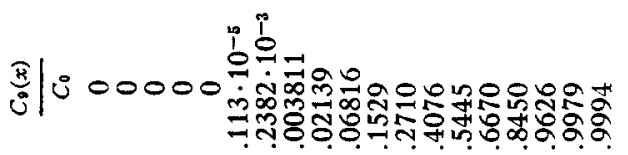

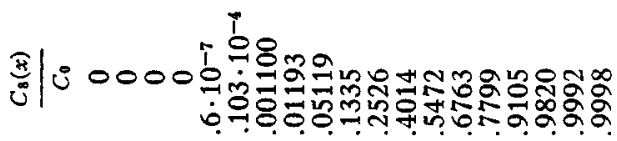

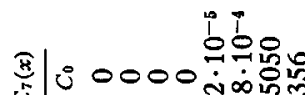

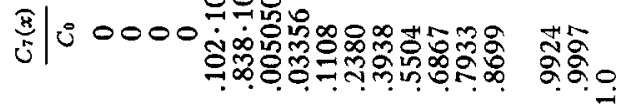

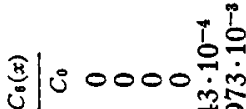

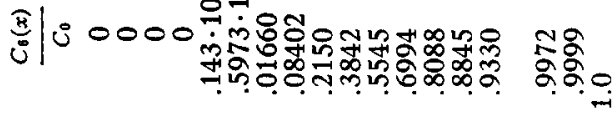

в

(1)

(a)

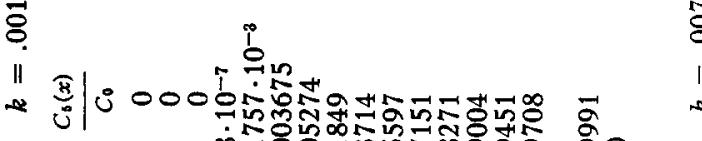

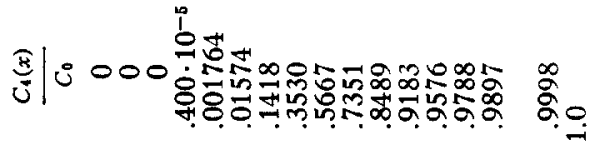

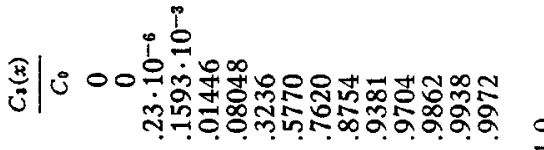

\section{bos}

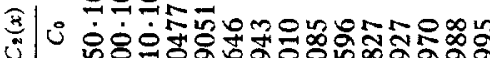

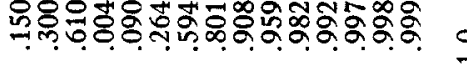

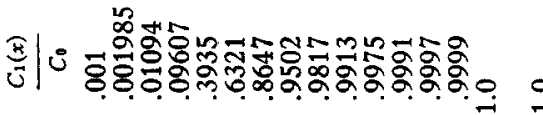

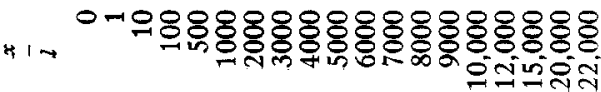

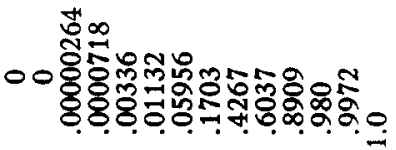

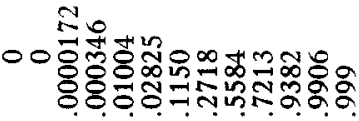

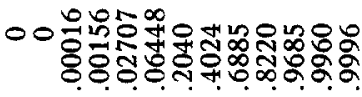

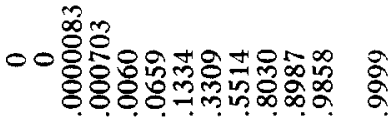

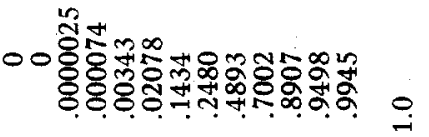

\&

00ํํㅇำ

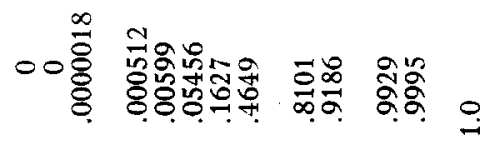

药

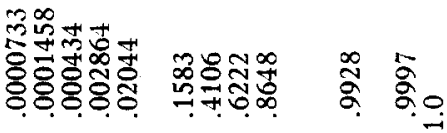

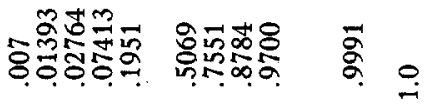

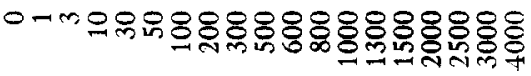


30

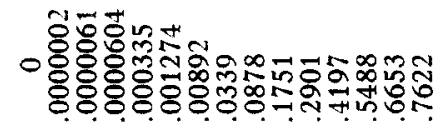

$\stackrel{8}{0} 10$

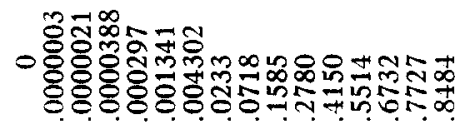

芯

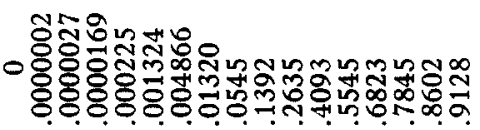

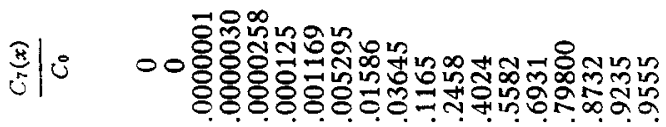

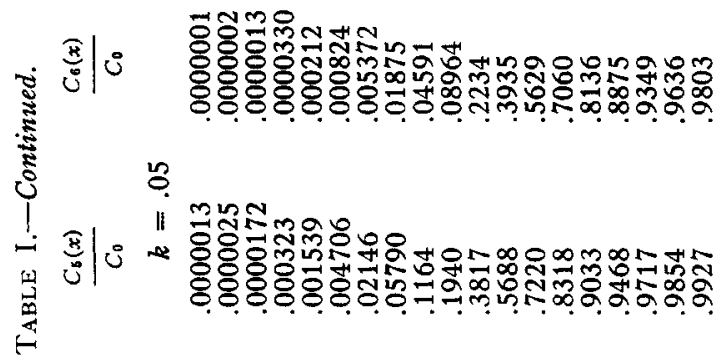

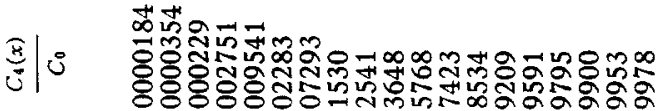

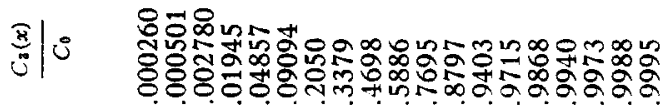

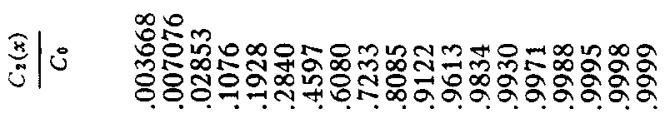

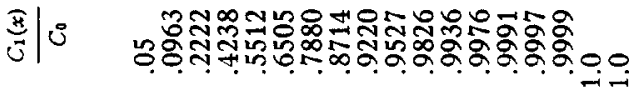




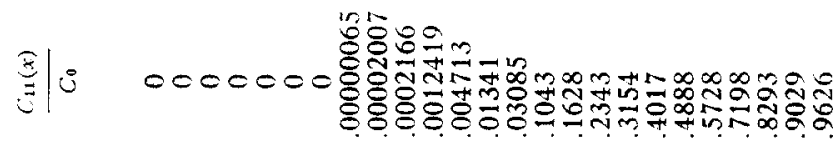

乳|: 000000 0

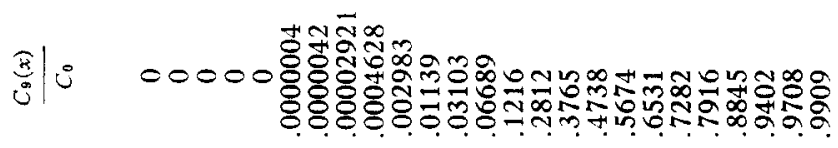

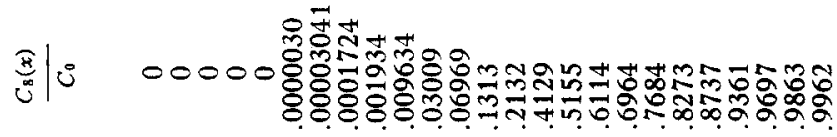

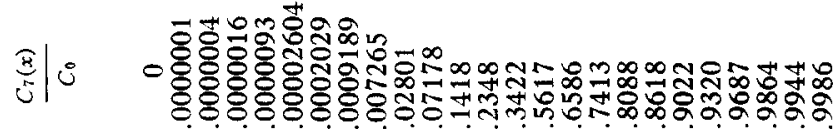

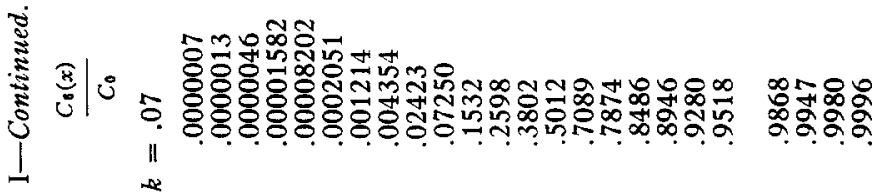

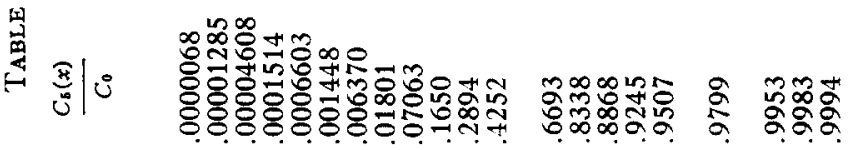

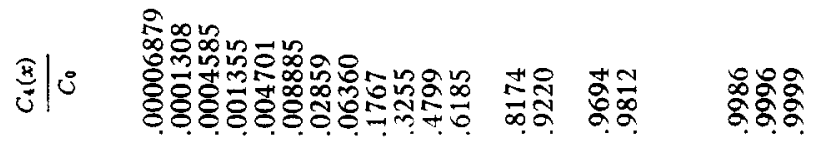

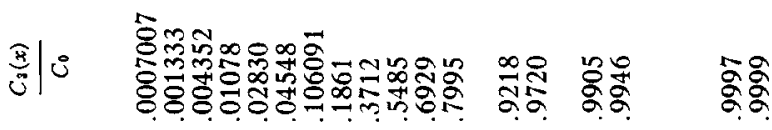

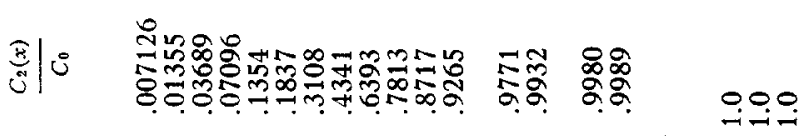

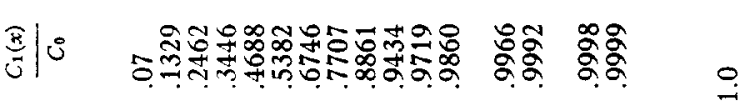

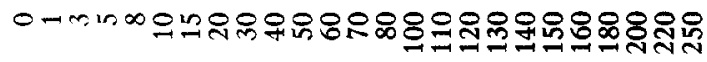




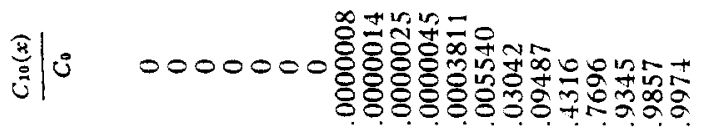

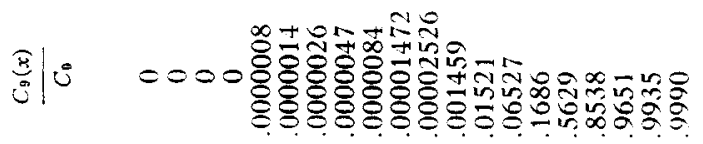

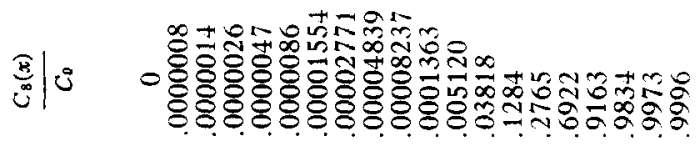

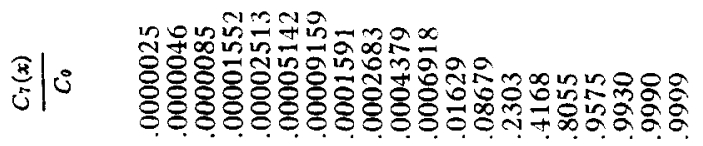

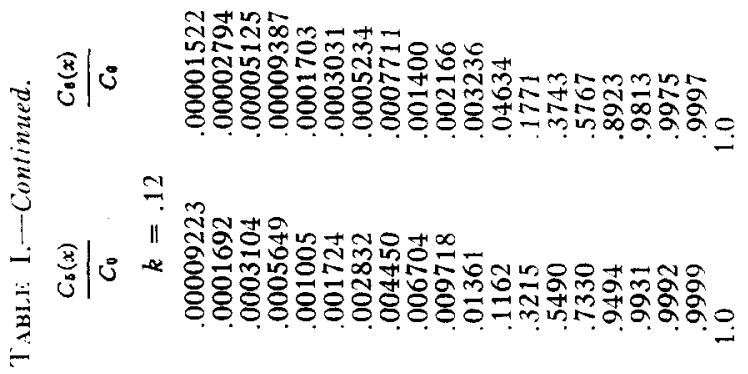

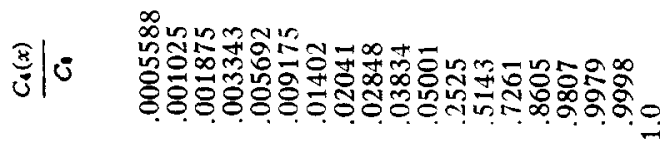

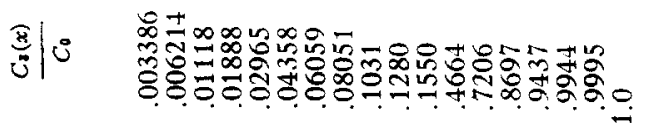

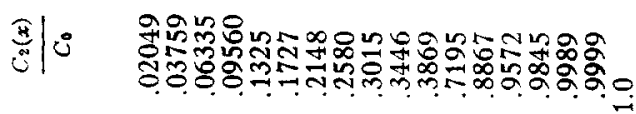

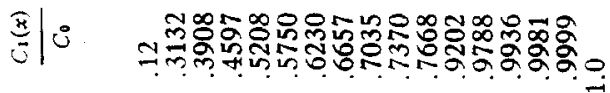


of $k$, ranging from $0.007,0.05,0.07$ to 0.12 are displayed successively in Figs. 3, 4, 5, and 6 .

These results of $C_{n}(x) / C_{0}$ are restricted to the infinite bar (that is, terminal zone freezing neglected) and supplement data now incorporated as standard matter (2). The validity of Eqs. 10 is thus established.

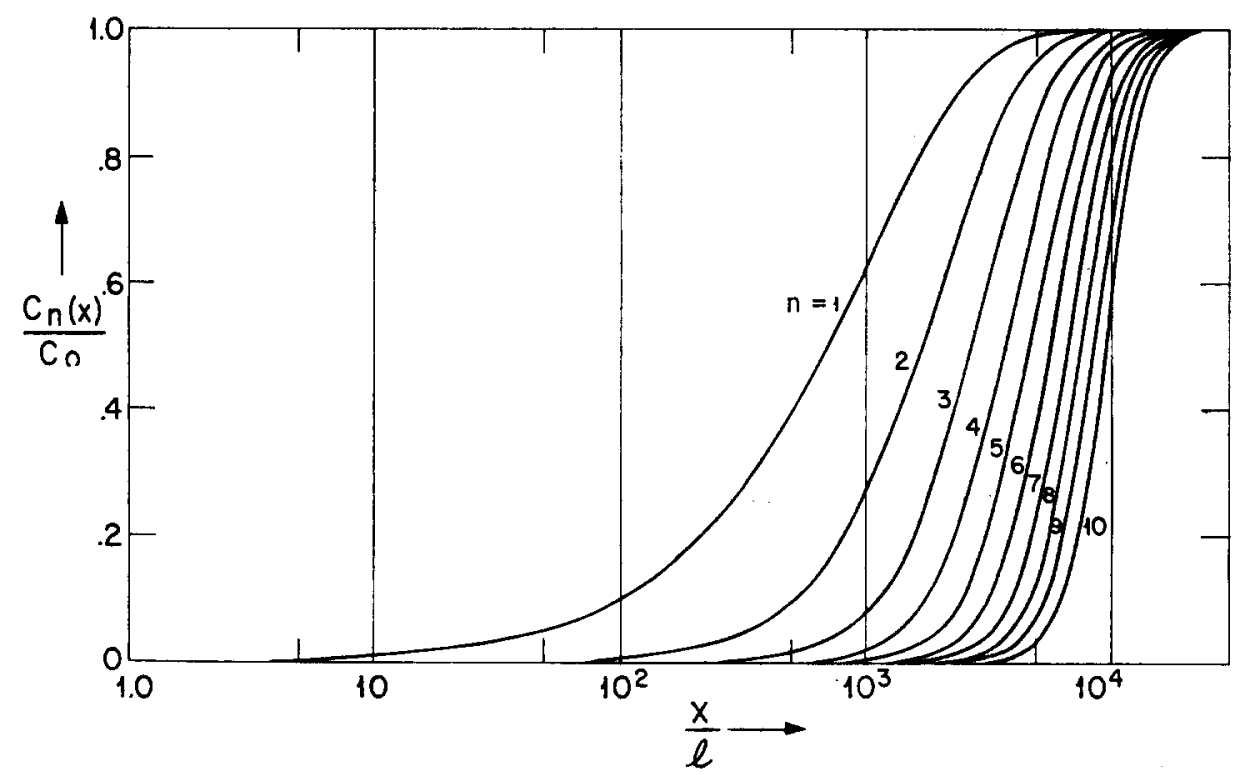

FIG. 1. Transformation of the solute distribution $C_{n}(x) / C_{0}$ by cascade zone refining. The parameter $n$ indicates stage of purification. The case $k=0.001$ showing approach to a limiting distribution as $n \rightarrow \infty$.

Incorporating the new findings in Eq. 4 produces

$$
P_{T}=\frac{l}{L}+\frac{(1-k)}{L} \int_{0}^{L-l} e^{-(k / l) x} \cdot \sum_{p=n}^{1} A_{p} \frac{\left(\frac{k}{l} x\right)^{n-p}}{(n-p) !} d x
$$

whence the purification factor may be written in the form

$$
\pi_{T}=1-\frac{l}{L}+\frac{(1-k)}{L} \cdot \frac{l}{k}\left[\frac{A_{n} B_{1}}{0 !}+\frac{A_{n-1} B_{2}}{1 !}+\frac{A_{n-2} B_{3}}{2 !}+\cdots \frac{A_{1} B_{n}}{(n-1) !}\right]
$$

wherein the $A_{n}$ are as stated in Eq. $10 c$ and now

$$
B_{n}=\left(\frac{k}{l}\right)^{n} \int_{0}^{L^{-l}} x^{n-1} e^{-(k / l) x} d x .
$$


CONCLUSION

The paramount objective of characterizing the zone-refinement operation by introduction of a purification factor has now been fulfilled. The investigation may be appreciated more fully by numerical

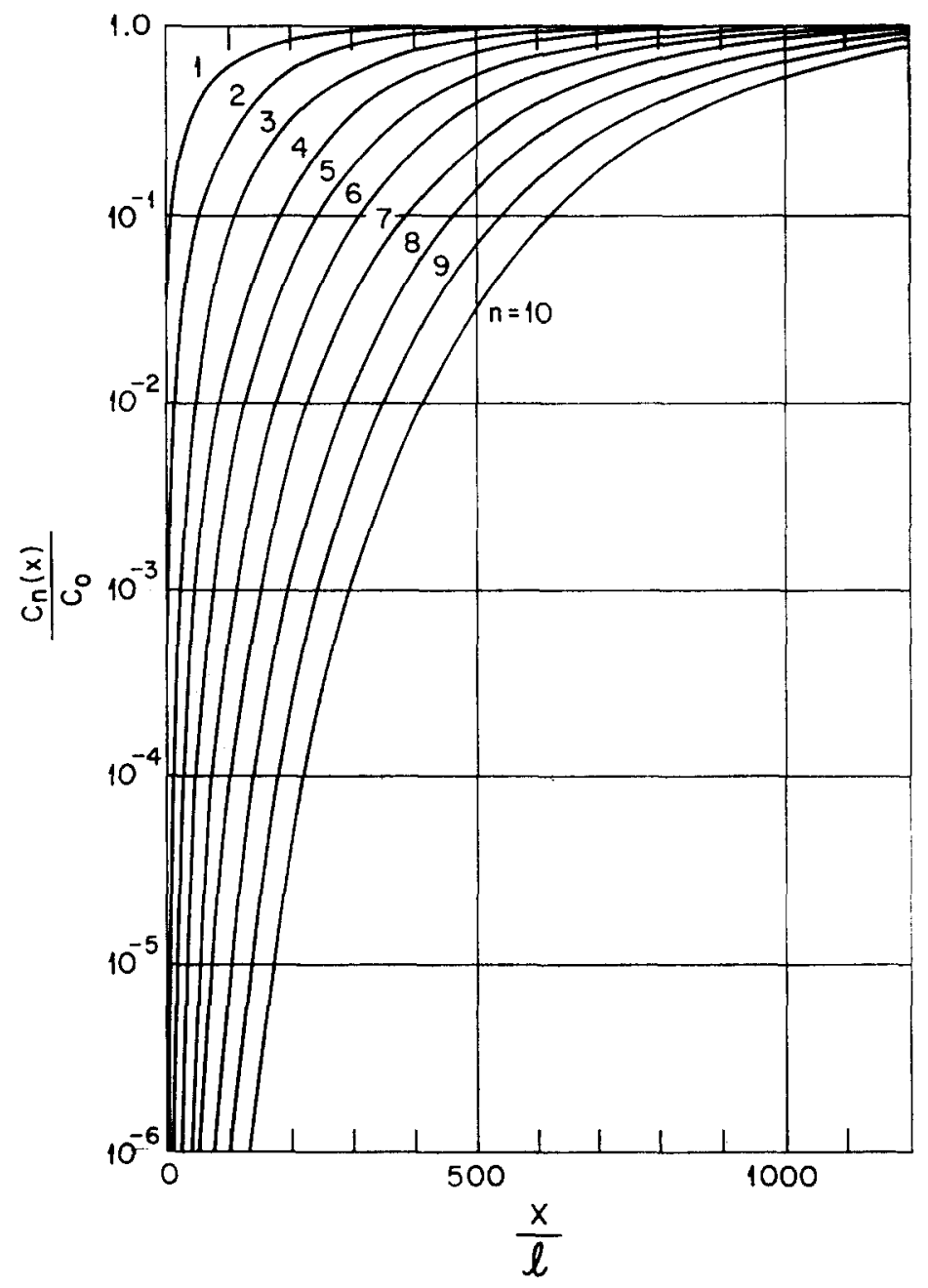

Fifr. 2. The case $k=0.001$ in the region of $\frac{x}{l} \rightarrow 0$. (Cf. Fig. 1.)

description of $\pi_{T}$ for the values of $k$ already embodied in the delineation of the solute distribution. Table II contains the $\pi_{T}$ behavior for three selected values of $l / L$, viz. $1 / 2,1 / 4$ and $1 / 10$. Finally, Figs. 7,8 and 9 depict the expected fall-off of the purification factor with increasing passes for the gamut of $k$ and $l / L$ indicated. 


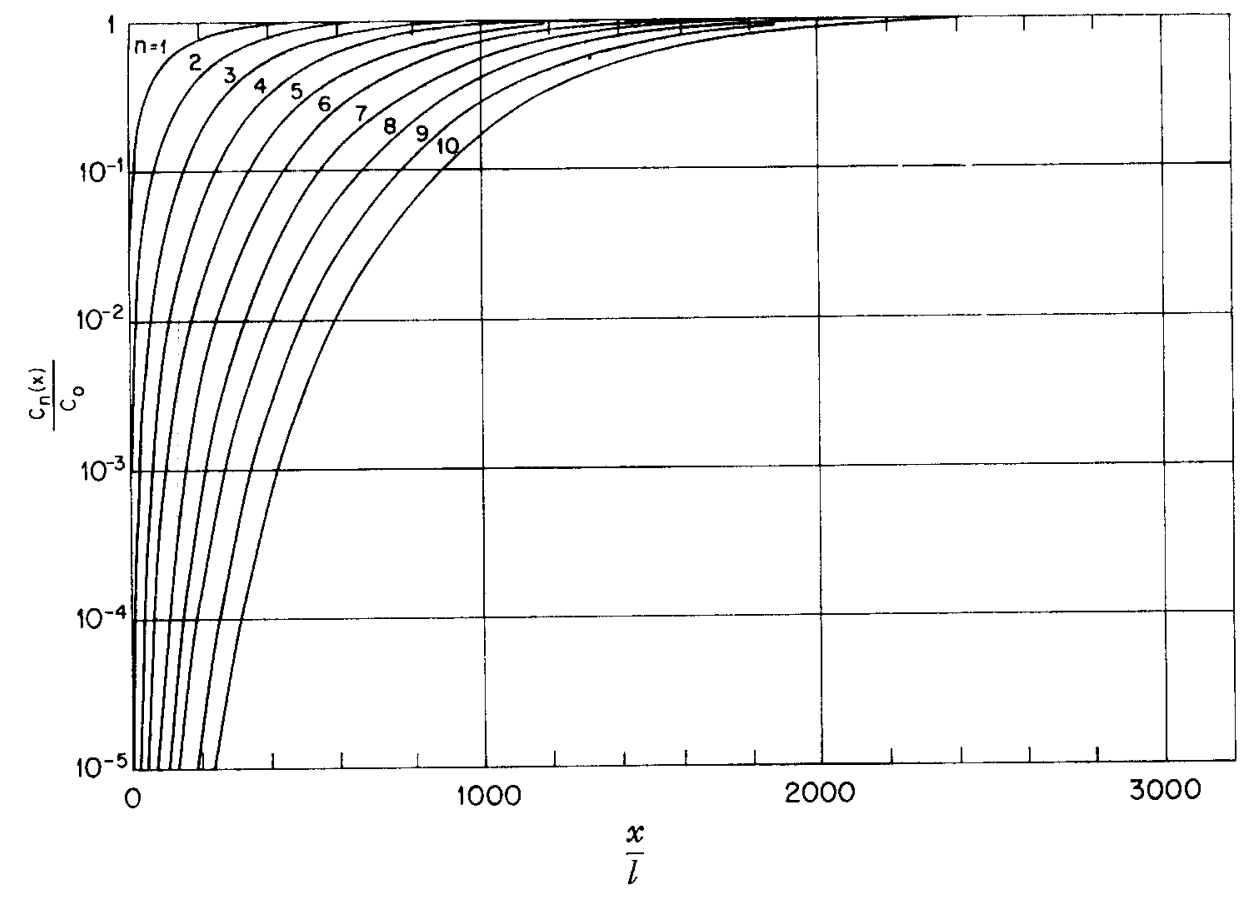

FIG. 3. The case $k=0.007$. (Cf. Fig. 1.)

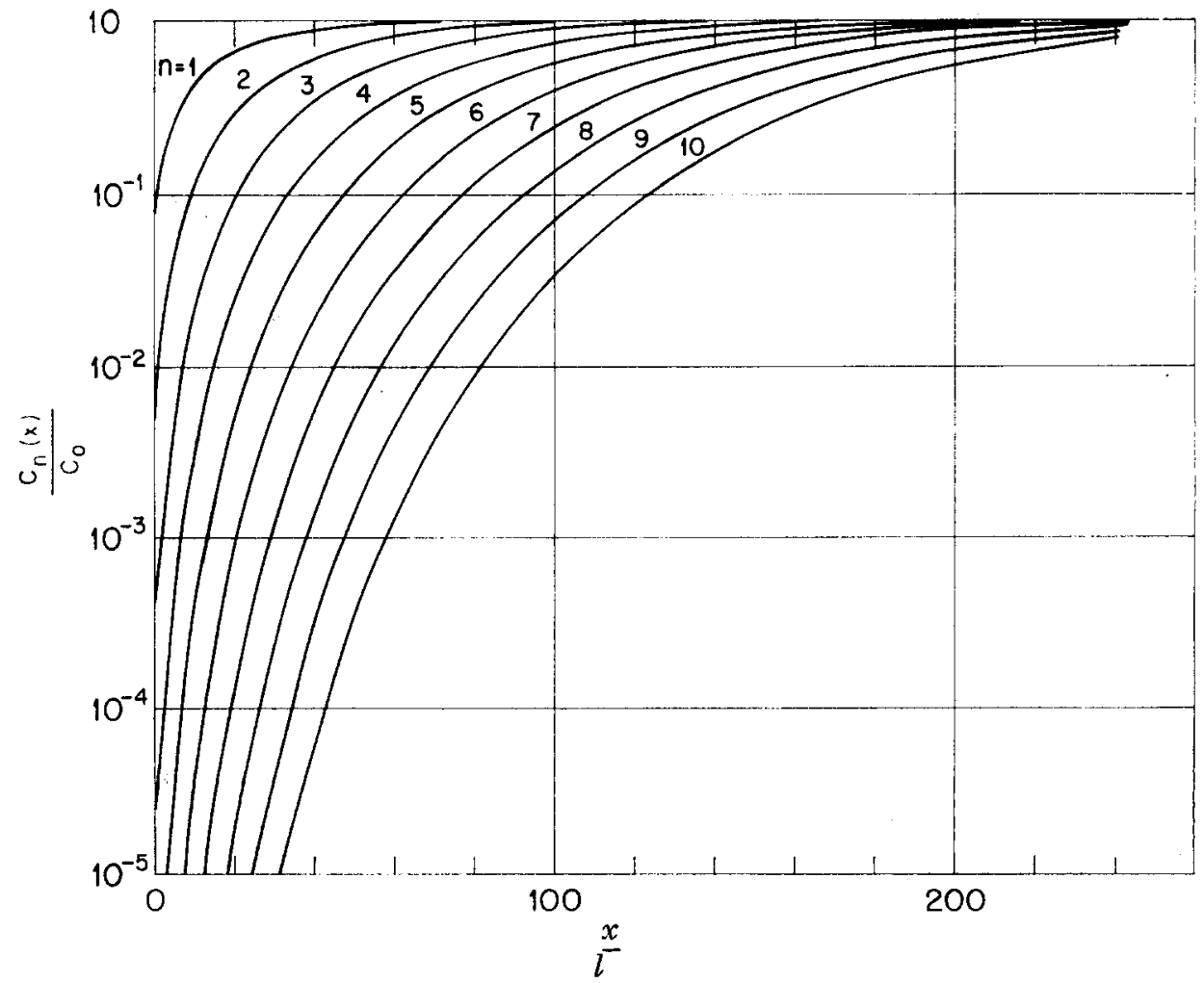

Frg. 4. The case $k=0.05$. (Cf. Fig. 1.) 
Nov., 1960.] Zone-Refining Purticication Factor

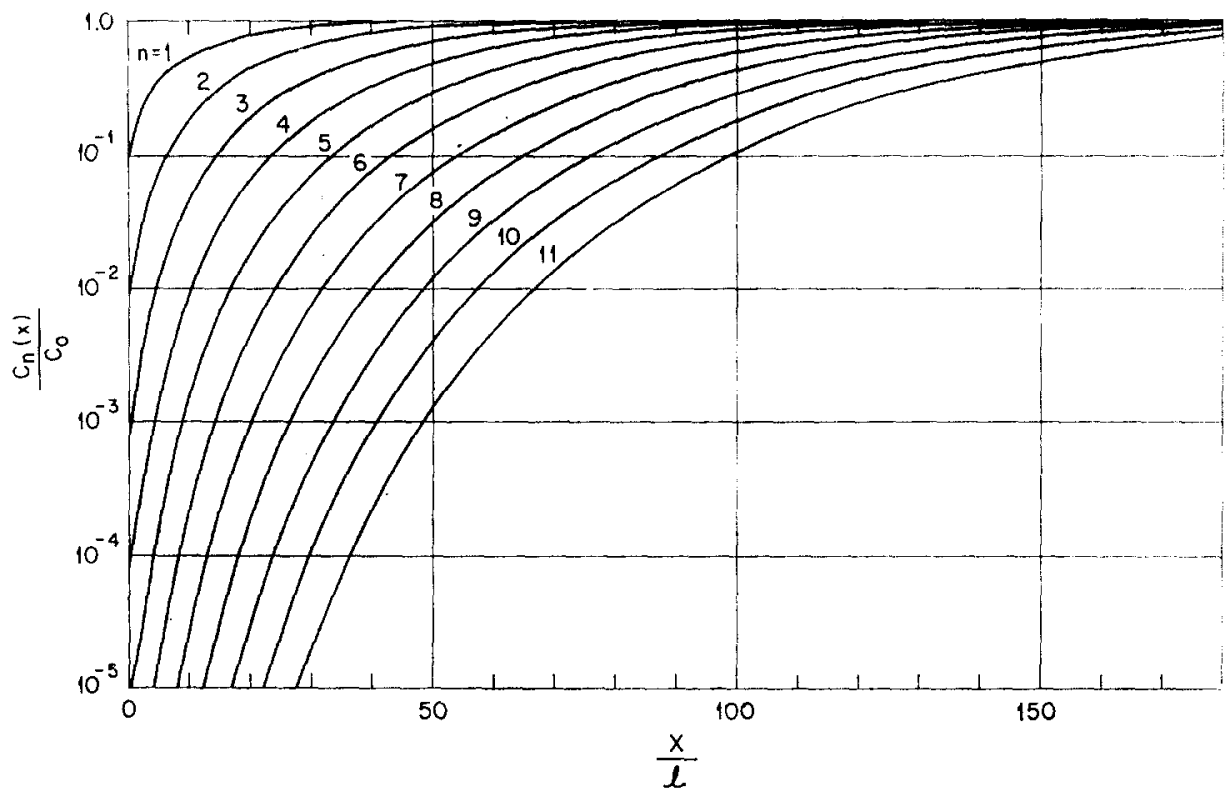

FIG. 5. The case $k=0.07$. (Cf. Fig. 1.)

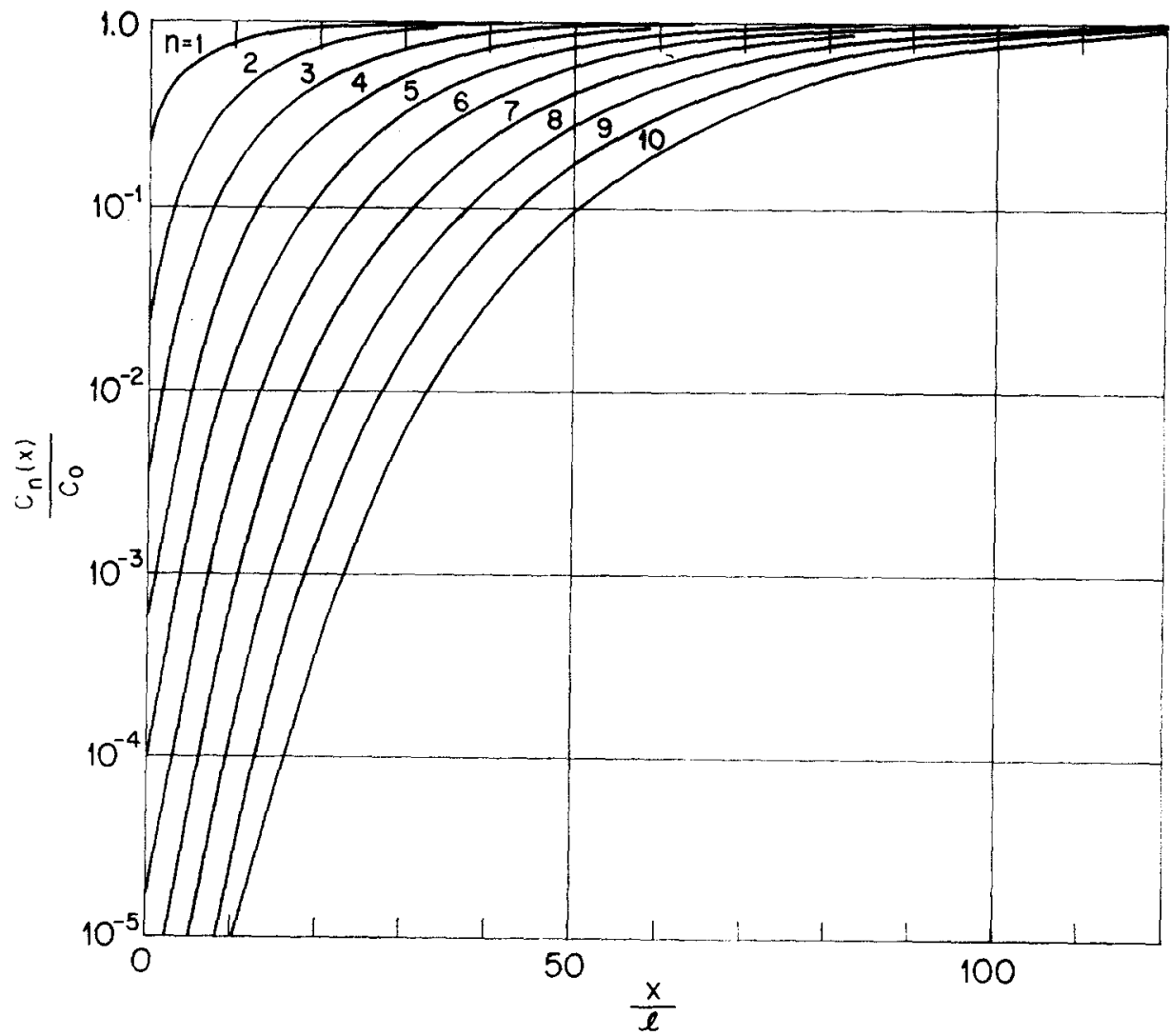

Fig. 6. The case $k=0.12$. (Cf. Fig. 1.) 


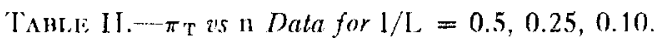

n

1

2

3

2

3

4

5

2

3

4

5

6

7

8

0052337

.0000528

.0000005

0

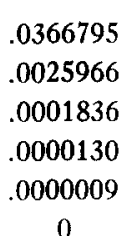

0

.0509018

.0050049

.0004914

.0000483

.0000047

.0000005

0

7

8

9

2

3

4

5

6

7

8

9

10

.0853749

.0141092

.0023283

.0003843

.0000634

.0000105

.0000017

.0000003

0 $l . t=0.25$

$k=.001$

.0018728

.0000034

0

$k=.007$

.0130154

.0001633

.0000018

0

.0338696

.00083117

.0000156

0000002

0

$k=.05$

.0883629

.0077118

.0005898

.0000428

.0000030

.0000002

0

.0058298

.0000174

0

.2114935

.0345802

.0044488

.0004804

.0000455

.0000039

.0000003

0

$k=.07$

.1208691

.2790148

.0145876

.0618266

.9015478

.0109057

.0001559

.0016245

.0000154

.0000015

.0000001

0

.0002129

.0000254

.0000028

.0000003

0

$$
k=.12
$$

.1957399

.4157034

.0392708

.1456024

.0069822

.0417406

.0011810

.0102595

.0001963

.0022383

.0000324

.0004459

.0000054

.0000830

.0000009

.0000148

0

.0000025

0 

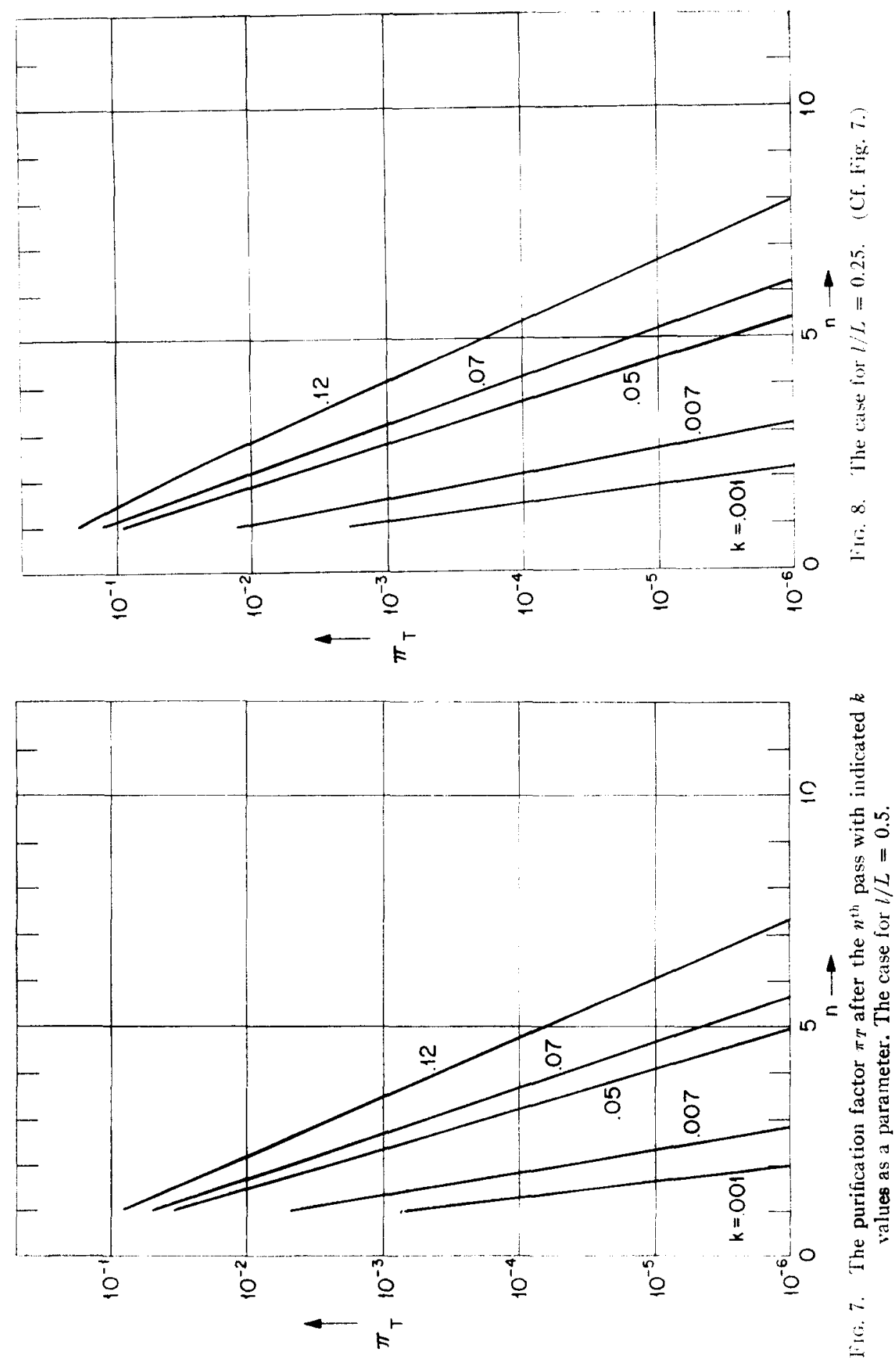


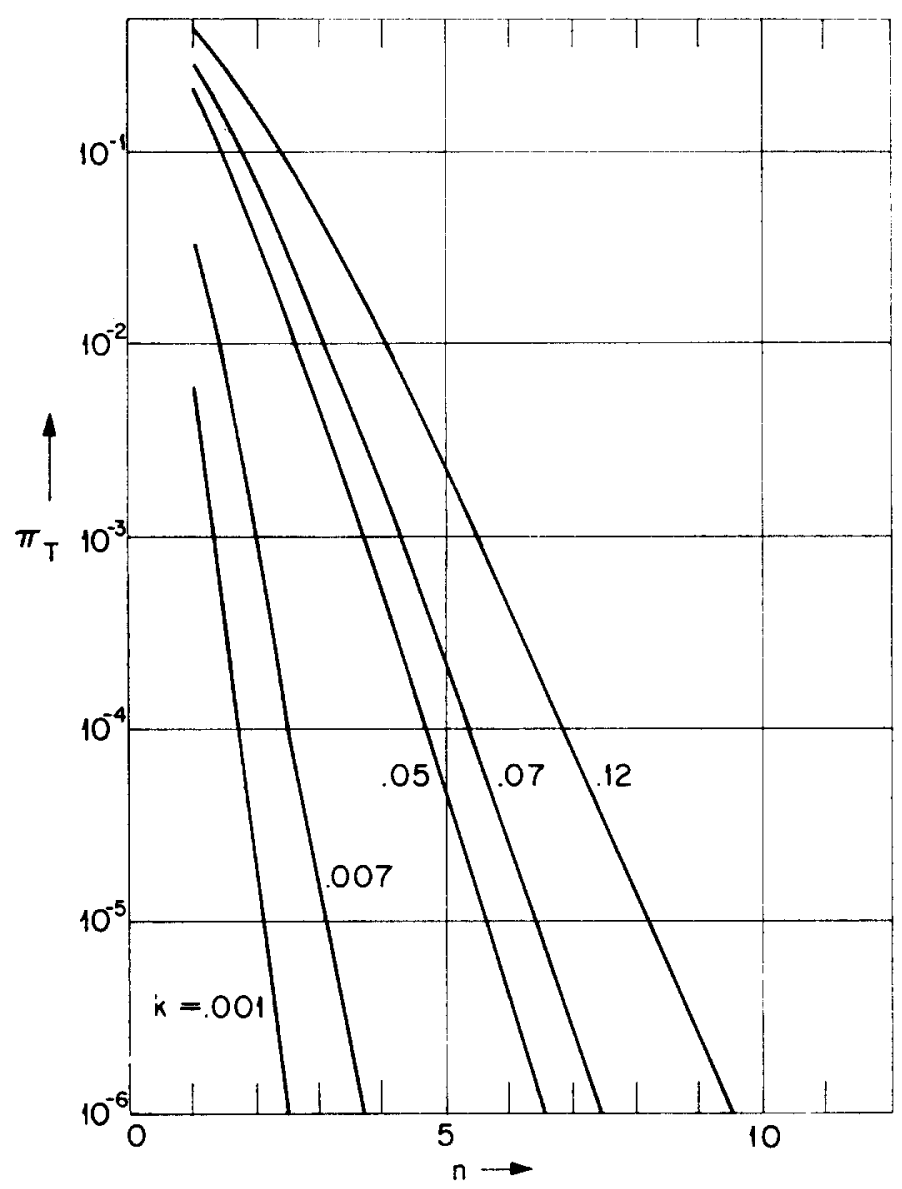

FIG. 9. The case for $l / L=0.10$. (Cf. Fig. 7.)

\section{APPENDIX}

The $Q_{n}(x)$ coefficients of Eq. $10 a$ assume successively the values:

$$
\begin{aligned}
Q_{1} & =1 \\
Q_{2} & =2-e^{-k}+\left(\frac{k}{l} x\right) \\
Q_{3} & =\left(2-e^{-k}\right)^{2}-k e^{-k}+\left(2-e^{-k}\right)\left(\frac{k}{l} x\right)+\left(\frac{k}{l} x\right)^{2} / 2 ! \\
Q_{1} & =\left(2-e^{-k}\right)^{3}-2 k e^{-k}\left(2-e^{-k}\right) \frac{k^{2}}{2} e^{-k}+\left[\left(2-e^{-k}\right)^{2}-k e^{-k}\right] \frac{k}{l} x \\
& \quad+\left(2-e^{-k}\right)\left(\frac{k}{l} x\right)^{2}+\left(\frac{k}{l} x\right)^{3} / 3 !
\end{aligned}
$$




$$
\begin{aligned}
& Q_{\dot{j}}=\left[\left(2-e^{-k}\right)^{4}-3 k e^{-k}\left(2-e^{-k}\right)^{2}-2 k^{2} e^{-k}\left(1-e^{-k}\right)-\frac{k^{3}}{6} e^{-k}\right] \\
& +\left[\left(2-e^{-k}\right)^{3}-2 k e^{-k}\left(2-e^{-k}\right)-\frac{k^{2}}{2} e^{-k}\right] \frac{k}{l} x \\
& +\left[\left(2-e^{-k}\right)^{2}-k e^{-k}\right]\left[\left(\frac{k}{l} x\right)^{2} / 2 !\right]+\left(2-e^{-k}\right)\left(\frac{k}{l} x\right)^{3} / 3 !+\left(\frac{k}{l} x\right)^{4} / 4 ! \\
& \bigcup_{6}=\left[\left(2-e^{-k}\right)^{5}-4 k e^{-k}\left(2-e^{-k}\right)^{3}-6 k^{2} e^{-k}\left(1-e^{-k}\right)^{2}\right. \\
& \left.+\frac{3}{2} k^{*} e^{\cdots k}-\frac{2}{3} k^{3} e^{-k}\left(1-2 e^{k}\right)-\frac{k^{4}}{24} e^{-k}\right] \\
& +\left[\left(2-e^{-k j}\right)^{t}-3 k e^{-k}\left(2-e^{-k}\right)^{2}-2 k^{2} e^{-k}\left(1-e^{\cdots k}\right)-\frac{k^{3}}{6} e^{-k}\right]\left(\frac{k}{l} x\right) \\
& +\left[\left(2-e^{-k}\right)^{2}-2 k e^{-k}\left(2-e^{-k}\right)-\frac{k^{2}}{2} e^{-k}\right]\left(\begin{array}{l}
k \\
i
\end{array}\right)^{2} / 2 ! \\
& +\left[\left(2-e^{-k}\right)^{2}-k e^{-k}\right]\left(\begin{array}{l}
k \\
l^{-} x
\end{array}\right)^{3} / 3 !+\left(2-e^{-k}\right)\left(\frac{k}{l} x\right)^{4} / 4 !+\left(\begin{array}{l}
k \\
l
\end{array}\right)^{5} / 5 !
\end{aligned}
$$

whence the recursion formula $(10 b)$ is deduced.

Applying the boundary condition at $x=0$, there obtains from Eqs. 7 and 9

$$
\frac{C_{n}(0)}{C_{0}}=\frac{k}{l} \int_{0}^{l} \frac{C_{n-1}(x) d x}{C_{n}}=1-(1-k) A_{n}
$$

or

$$
A_{n}=\left(1-k_{i}^{-1}\left(1-\frac{C_{n}(0)}{C_{n}}\right)\right.
$$

In this manner Eqs. $10 c$ and $10 d$ follow with the lirst few coefficients identified as

and -

$$
A_{1}=1, \quad A_{2}=2-e^{-k}, \quad A_{3}=\left(2-e^{-k}\right)^{2}-k e^{-k}
$$

$$
S_{1}=e^{-k}-1, \quad S_{2}=e^{-k}(k+1)-1, \quad S_{3}=e^{-k}\left(k^{2}+2 k+2\right)-2 .
$$

\section{REFERENCES}

(1) L. Gold, J. Phys. Sac. Japan, Vol. 14, p. 386 (1959).

(2) W. G. Pfann, "Zone Melting," New York, John Wiley \& Sons, Inc., 1958.

(3) W. G. Pfans, Trans. A.I.M.M.E., Vol. 194, p. 747 (1952). 